\title{
MELTING AT THE LIMIT OF SUPERHEATING
}

\author{
Sheng-Nian Luo, ${ }^{1}$ Thomas J. Ahrens, ${ }^{2}$ and Damian C. Swift ${ }^{1}$ \\ ${ }^{1}$ P-24 Plasma Physics, Los Alamos National Laboratory, Los Alamos, NM 87545 \\ ${ }^{2}$ Seismological Laboratory, California Institute of Technology, Pasadena, CA 91125
}

\begin{abstract}
Theories on superheating-melting mostly involve vibrational and mechanical instabilities, catastrophes of entropy, volume and rigidity, and nucleation-based kinetic models. The maximum achievable superheating is dictated by nucleation process of melt in crystals, which in turn depends on material properties and heating rates. We have established the systematics for maximum superheating by incorporating a dimensionless nucleation barrier parameter and heating rate, with which systematic molecular dynamics simulations and dynamic experiments are consistent. Detailed microscopic investigation with large-scale molecular dynamics simulations of the superheating-melting process, and structure-resolved ultrafast dynamic experiments are necessary to establish the connection between the kinetic limit of superheating and vibrational and mechanical instabilities, and catastrophe theories.
\end{abstract}

\section{INTRODUCTION}

Melting and freezing as first-order phase changes and their related kinetics, are of ubiquitous theoretical and experimental interest in condensed matter physics, materials science and engineering, geophysics and planetary sciences.[1] Metastable superheating and undercooling are inherent in melting and freezing processes. Determining the degree to which a solid can be superheated and a liquid undercooled, is a fundamental and challenging issue. Experimental investigation of the maximum superheating is particularly difficult due to the existence of heterogeneous nucleation sites (e.g. free surfaces and defects), and the difficulty in achieving high heating rates while making sensible measurements. Theoretical efforts in understanding superheating-melting have been seriously undermined by the paucity in superheating data. Molecular dynamics (MD) simulations have been utilized to probe melting and freezing processes at atomic level, and serve an important complementary approach to theoretical and experimental techniques.

Previous superheating-melting theories[1] in- cluding Lindemann and Born's criteria, orderdisorder transition, catastrophes of entropy, volume and rigidity, and nucleation-based kinetic models are briefly reviewed. We present certain details on the recently developed systematics for the maximum superheating and undercooling.[1] Experimental, theoretical and simulation directions for future investigations of superheatingmelting process are presented.

\section{SUPERHEATING-MELTING THEORIES}

Solids differ distinctly from liquids in both their long-range order and ability to resist shearing. The definitions and criteria for melting mostly involve vibrational and mechanical instabilities and order-disorder transitions.[1] Lindemann's vibrational criterion[1] states that melting occurs at the onset of an instability when the atomic displacements (e.g. the root-meansquared displacements) during thermal vibrations exceed a certain threshold. Born's mechanical criterion[1] states that the stability against shearing stress vanishes (e.g. for cubic lattice $c_{44}=0$ where $c_{44}$ is the elastic constant in 
Voigt's notation) and such shearing instability is essentially melting. Melting is also interpreted as structure transition from order to disorder as proposed by Lennard-Jones and Devonshire.[1] Such a order-disorder transition is arguably attributed by Cahn[1] to the spontaneous production of intrinsic lattice defects.

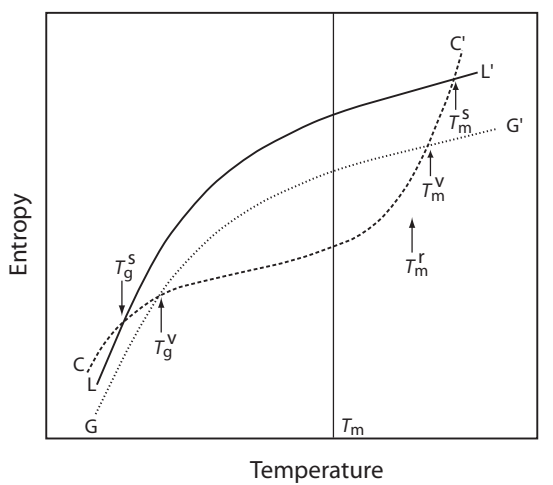

FIGURE 1. Schematic of entropy vs. temperature: A hierarchy of catastrophes as a succession of stability limits for the crystalline state. After Tallon.[1]

These instabilities in thermal vibration and resistance to shearing, and breakdown of longrange order are definitions of melting which inadequately describe the mechanism of melting, i.e. the kinetics of melting. Similarly, equilibrium thermodynamics simply states that liquid has lower Gibbs free energy than solid above melting temperature, $T_{m}$. Without considering nucleation process, catastrophes of certain physical quantities (e.g. molar entropy $s$, molar volume $v$ and rigidity $r$ ) are employed by Tallon[1] to define a hierarchy of the limit of superheating (Fig. 1). The entropy of solid (along $C C^{\prime}$ ) intersects that of liquid $\left(L L^{\prime}\right)$ at $T_{g}^{s}$ and $T_{m}^{s}$ $\left(T_{g}^{s}<T_{m}^{s}\right)$. According to Kauzmann, [1] the glass transition preempts at the catastrophe point $T_{g}^{s}$, an undercooling state. Fecht and Johnson[1] thus proposed that melting preempts at $T_{m}^{s}$, the counterpart of $T_{g}^{s}$. The maximum superheating occurs at the $T_{m}^{s}$ where the entropies of solid and liquid are equal. By correcting the liquid entropy for communal entropy of the liquid (e.g. $R \ln 2$ for certain monatomic systems where $R \equiv$ gas constant), the entropy line $G G^{\prime}$ intersects that of solid at $T_{g}^{v}$ and $T_{m}^{v}\left(T_{g}^{v}<T_{m}^{v}\right)$ where the molar volumes of liquid (glass) and crystal are equal. Beyond $T_{m}^{v}$, liquid would become denser for normal materials, thus melt preempts at $T_{m}^{v}$. Isochoric melting defines a smaller superheating $T_{m}^{v}$ at the volume catastrophe. Tallon further argued that rigidity instability occurs where the density of the superheated crystal equal to that of the liquid at the freezing point. This defines a superheating state $T_{m}^{r}$ at the catastrophe of rigidity. The hierarchy of catastrophes of a succession of stability limits for crystalline state is elastic rigidity $\left(T_{m}^{r}\right)$, volume $\left(T_{m}^{v}\right)$ and entropy $\left(T_{m}^{s}\right) \cdot[1]$

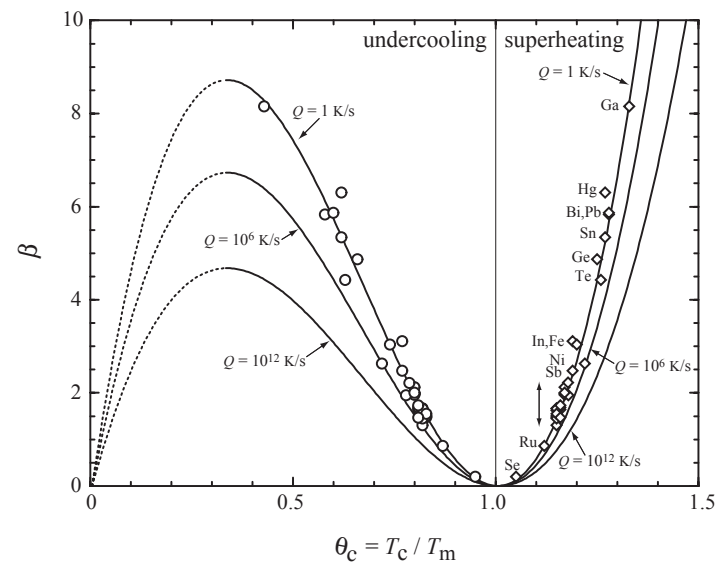

FIGURE 2. The systematics[1] of maximum superheating and undercooling for elements: $\beta=\left(A_{0}-b \lg Q\right) \theta_{c}(1-$ $\left.\theta_{c}\right)^{2}$. Circles are experimental value of undercooling at cooling rate $Q \sim 1 \mathrm{~K} / \mathrm{s}$, and diamonds are calculated superheating at $Q \sim 1 \mathrm{~K} / \mathrm{s}$. Solid and dotted curves are plots with $Q=1,10^{6}$ and $10^{12} \mathrm{~K} / \mathrm{s}$, respectively. Dotted curves denote the undercooling portions for $\theta_{c}=0-1 / 3$. The maximum of $\beta$ for undercooling occurs at $\theta_{c}=1 / 3$ for each $Q$. The elements within the double-headed arrow are $\mathrm{Ti}, \mathrm{Al}, \mathrm{Au}, \mathrm{Cu}, \mathrm{Hf}, \mathrm{Cd}, \mathrm{Pd}, \mathrm{Ag}, \mathrm{Co}, \mathrm{Pt}, \mathrm{Ta}, \mathrm{Rh}, \mathrm{Zr}$, $\mathrm{Mn}, \mathrm{Si}, \mathrm{Sb}, \mathrm{Ni}$, In and $\mathrm{Fe}$ in $\beta$-increasing order.

The concept of defining the limit of superheating by a hierarchy of catastrophes has clear physical implications but is oversimplified, and cannot be regarded as universal and is of little practical use. A large range of superheating is predicted by the catastrophes.[1] Furthermore, nucleation is inherent in melting and freezing process and depends on an integral of several physical parameters such as solid-liquid interfacial energy $\left(\gamma_{s l}\right)$, heat of fusion per unit volume 
$\left(\Delta H_{m}\right)$ and $T_{m}$, and heating rate $Q$. An appropriate estimation of superheating should be nucleation-based. Given $\gamma_{s l}$ from undercooling experiments and assuming nucleation rate $I=1$ $\mathrm{s}^{-1} \mathrm{~cm}^{-3}, \mathrm{Lu}$ and $\mathrm{Li}[1]$ predicted less amount of superheating than Tallon's hierarchy.[1] Rethfelder et al.[1] estimated superheating assuming a critical volume of nucleation is formed during a given time scale. Although both studies are nucleation-based, their studies did not reveal the systematic nature of melting, and heating rate was not included.

We recently developed a framework[1] for the systematics of maximum superheating and undercooling which are based on undercooling experiments and classical nucleation theory, and incorporate heating rates. Systematic MD simulations and dynamic melting experiments demonstrate significant consistency with the systematics. Next we discuss the systematics for maximum superheating.

\section{SYSTEMATICS FOR MAXIMUM SUPERHEATING}

The technical challenges of achieving homogeneous nucleation in melting experiments limit the amount of data of superheating which in turn limits development of a practical superheatingmelting theory. But a significant number of freezing experiments have been conducted where appreciable undercooling has been observed with homogeneous nucleation of crystals in liquids. As $\gamma_{s l}, \Delta H_{m}$ and $T_{m}$ are common to both melting and freezing, undercooling experiments would allow us to make predictions on superheating.

Based on classical nucleation theories, the nucleation rate[1] for both melting and freezing can be expressed and approximated as

$$
I=M(m, T) \exp \left\{-\frac{\Delta G_{c}}{k T} g(\phi)\right\} \approx I_{0} f(\beta, \theta)
$$

where $M$ is a function of material properties $(m)$ and temperature $(T) . \Delta G_{c}$ is the critical Gibbs free energy for nucleation, $k$ Boltzmann's constant, and $g(\phi)$ a geometrical factor depending on the wetting angle $\phi$ of a heterogeneous nucleant. For homogeneous nucleation, $g(\phi)=1$, the case assumed in the following discussions. $I_{0}$ is a constant prefactor.[1] We define the energy barrier for nucleation, $\beta$, as a dimensionless quantity,

$$
\beta\left(\gamma_{s l}, \Delta H_{m}, T_{m}\right)=\frac{16 \pi \gamma_{s l}^{3}}{3 \Delta H_{m}^{2} k T_{m}}
$$

and the reduced temperature as $\theta=T / T_{m}$, and

$$
f(\beta, \theta)=\exp \left\{-\frac{\beta}{\theta(\theta-1)^{2}}\right\}
$$

Thus, the nucleation process is essentially dependent on $\beta$ and $\theta$. We denote the maximum superheating and undercooling as $\theta_{c}=T_{c} / T_{m}$. Previous undercooling experiments yielded values of $\theta_{c}^{-}$(- denotes undercooling), $\gamma_{s l}, \Delta H_{m}$ and $T_{m}$ (thus $\beta$ ) for elements and compounds.[1] $\theta_{c}$ obviously depends on heating (or cooling) rate $(Q)$. Normally the reported experimental values of $\theta_{c}^{-}$ are for $Q_{0} \sim 1 \mathrm{~K} / \mathrm{s}$.

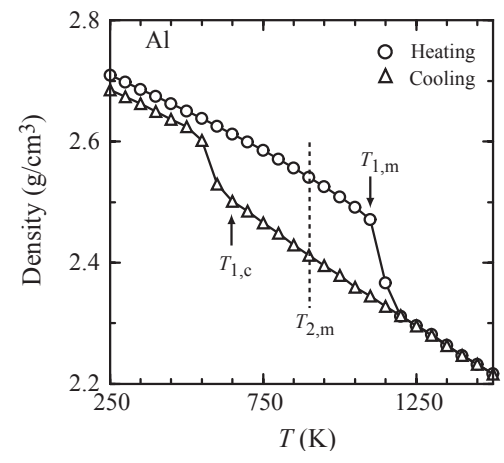

FIGURE 3. Typical single- and two-phase MD simulations of the melting and refreezing behavior: density vs. T. A complete hysteresis of density forms during stepped heating-cooling process for Al. $T_{1, m}$ and $T_{1, c}$ are the single-phase melting and freezing temperature at the superheated and undercooled states, respectively. $T_{2, m}$ is the equilibrium melting temperature from the two-phase simulations. Thus, $\theta_{c}^{+}=T_{1, m} / T_{2, m}$ and $\theta_{c}^{-}=T_{1, c} / T_{2, m}$.

As $\beta$ is common to both melting and freezing, values of $\beta$ and $\theta_{c}^{-}\left(Q_{0}\right)$ allow us to predict the maximum undercooling $\theta_{c}^{-}(Q)$ and superheating $\theta_{c}^{+}(Q)$ at certain cooling and heating rate $Q$. For steady-state homogeneous nucleation of crystals from liquid (or melt in solid), Kelton[1] proposed 
that the probability $x$ for a given amount of parent phase of volume $v$ containing no new phase under certain cooling (or heating) rate $Q$ is

$$
x=\exp \left\{ \pm \frac{v T_{m} I_{0}}{Q} \int_{\theta_{c}}^{1} f(\beta, \theta) d \theta\right\}
$$

where + refers to superheating and - to undercooling. The parameters for undercooling experiments at $Q \sim 1 \mathrm{~K} / \mathrm{s}$, such as $\gamma_{s l}, \Delta H_{m}, T_{m}$ (thus $\beta$ ), and $v$ can be regarded as equal to those for superheating and undercooling at different heating and cooling rates. By assuming $x$ and $I_{0}$ is approximately equal for the undercooling and superheating cases, the maximum superheating and undercooling under any $Q$ can be calculated from experimental value of $\theta_{c}^{-}\left(Q_{0}\right)$ (Fig. 2). The numerical relationship[1] between $\beta, \theta_{c}$ and $Q$ is fitted as

$$
\beta=\left(A_{0}-b \lg Q\right) \theta_{c}\left(\theta_{c}-1\right)^{2}
$$

where $A_{0}=59.4, b=2.33$, and $Q$ is normalized by $Q_{0}=1 \mathrm{~K} / \mathrm{s}$. Eq. (5) is referred to as the $\beta-\theta_{c}-Q$ systematics for the maximum superheating and undercooling.

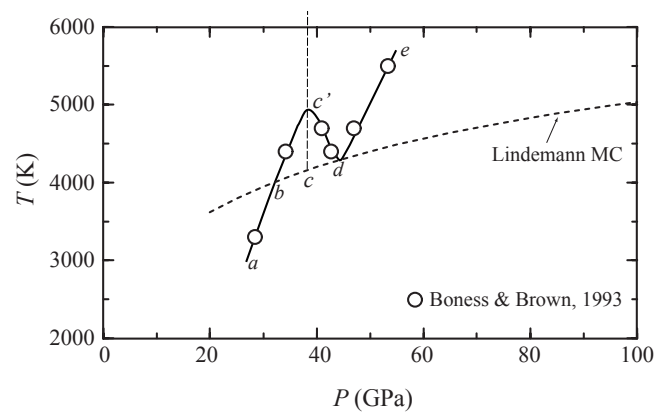

FIGURE 4. Shock-melting experiments on $\mathrm{CsBr}[1]$ demonstrate simultaneous drop in shock temperature and sound-speed (not shown), signaling melting of shocked crystal at higher shock pressures than $P_{c}$ (the long dashed curve). Solid curves indicate the Hugoniot states. The dashed curve is the Lindemann melting curve (MC).[1] $b c^{\prime}$ segment denotes superheated states.

The $\beta-\theta_{c}-Q$ systematics for maximum superheating and undercooling are empirical in nature. An independent verification is MD simula- tions of superheating and undercooling. Superheating was observed previously in a few studies.[1] We conducted systematic MD simulations with single- and two-phase techniques on $f c c$ metals (Al, Ni, Cu, Rh, Pd, Ag, Ir, Pt, Au and $\mathrm{Pb}$ ) and Be.[1] A typical example is shown in Fig. 3 for Al where superheating and undercooling can be determined. Current and previous simulations yielded values consistent with the $\beta-\theta_{c}-Q$ systematics at $Q \sim 10^{12} \mathrm{~K} / \mathrm{s}$. Thus the empirical systematics are validated at atomic level from MD simulations.[1]

Superheating has been observed in planar impact experiments with light-gas gun loading and intense laser irradiation on silicates, alkali halides and metals.[1] A representative example of shock-induced superheating $\left(Q \sim 10^{12} \mathrm{~K} / \mathrm{s}\right)$ is shown in Fig. 4 for CsBr. Experimental superheating values compare favorably to the prediction of the systematics.[1]

\section{DISCUSSION}

We have established the $\beta-\theta_{c}-Q$ systematics for the maximum superheating and undercooling consistent with MD simulations and dynamic experiments. Future experimental efforts will employ in-situ structure-resolved melting experiments with exploding wire and shockwave techniques.[2] MD simulations and theoretical efforts are needed to establish a universal relationship between kinetic limit of superheating and various definitions of melting, and catastrophe theories. The effects of heterogeneous nucleation sites at high heating rates, low dimensions and anisotropy, are also of interest.

\section{ACKNOWLEDGMENTS}

This work has been supported by U.S. NSF Grant EAR-0207934. S.-N. Luo is sponsored by a Director's Post-doctoral Fellowship at Los Alamos National Laboratory (P-24 and EES-11).

\section{REFERENCES}

1. Luo, S.-N., and Ahrens, T.J., Appl. Phys. Lett. 82, 1836 (2003); Luo, S.-N. and Ahrens, T.J., Phys. Earth Planet. Int. (in press) (2003); Luo, S.-N., Ahrens, T.J., Çağın, T., Strachan, A., Goddard III, W.A., and Swift, D.C., Phys. Rev. B 68, 134206 (2003), and references therein.

2. Luo, S.-N., Swift, D.C., Tierney, T., Xia, K., Tschauner, O., and Asimow, P.D., this conference. 